\title{
Yttrium-90 Radioembolization For Unresectable Chemo Refractory Breast Cancer Liver Metastases
}

\author{
Jinendra Satiya ${ }^{* 1}$, Kapil Gupta ${ }^{1}$ and Aviv Katz DO ${ }^{2}$ \\ ${ }^{1}$ Internal Medicine Residency Program, University of Miami Miller School of Medicine, USA \\ ${ }^{2}$ Digestive Diseases, JFK Medical Center, USA
}

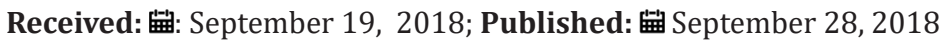

*Corresponding author: Jinendra Satiya, Internal Medicine Residency Program, University of Miami Miller School of Medicine Palm Beach Regional GME Consortium, West Palm Beach, FL, United States

\section{Introduction}

Yttrium-90 microsphere radioembolization with (90)Y microspheres is a locoregional radiation therapy employed for unresectable hepatic neoplasms by permanently implanting (90)Y-containing microspheres into tumors via the hepatic artery. 1 Non-target delivery of (90)Y microspheres can result in gastrointestinal (GI) symptoms. We present the case of a woman with ischemic gastropathy as a result of Y-90 therapy targeted for hepatic metastasis from invasive ductal carcinoma of the breast [1].

\section{Case Description}

This is a 64year-old female with breast cancer with isolated hepatic metastasis. As a result, she underwent chemoembolization of this hepatic lesion. Days later she developed left upper quadrant abdominal pain associated with nausea and vomiting. Several days of empiric proton-pump inhibitors and antiemetics offered no benefit. Upper endoscopy demonstrated a large wedged shaped area of severe gastric erythema and ulceration extending from the cardia esophageal junction to the incisura with linear margins. The temporal relationship of the Y90 radioembolization coupled with the endoscopic findings were suggestive of ischemic gastropathy. Pathology confirmed results ulcerated gastric mucosa with basophilic beads in vascular spaces, consistent with Y-90 radioembolization beads. Immunohistochemistry was negative for $\mathrm{H}$. pylori. These findings suggest gastropathy due to radioembolization.

\section{Discussion}

Radioembolization using yttrium-90 microspheres (Y-RE) is a novel modality of treatment for breast cancer liver metastases
(BCLM) patients in whom other locoregional and systemic treatment options have failed. The etiology of persistent gastric ulcerations in patients presenting months after radioembolization treatment remains unclear. Postradioembolization syndrome, including fatigue, abdominal pain, nausea, vomiting, anorexia, or fever is fairly common and is seen in upto $70 \%$ patients undergoing radioembolization. Diffusion-weighted magnetic resonanceimaging is superior to PET/CT in assessing treatment response and can be used to guide further management in these patients. Patients with left lobe tumors adjacent to the stomach do not exhibit radiation effects following radioembolization [2]. Studies have shown Y-RE to be a safe and effective treatment option for BCLM patients and for those with hepatic metastasis from colorectal cancer. Studies investigating the use of Y-RE combined with systemic therapy are required.

\section{Conclusion}

(90)Y microsphere radioembolization is a generally welltolerated treatment for unresectable hepatic tumors. It can also be performed for metastases from colorectal cancer. The antitumoral effect is supported by imaging and tumor marker responses. Most common complications include cholecystitis, postradioembolization syndrome and elevated liver chemistries.

\section{References}

1. Khajornjiraphan N, Thu NA, Chow PKH (2015) Yttrium-90 Microspheres: A Review of Its Emerging Clinical Indications. Liver Cancer 4(1): 6-15.

2. Murthy R, Kamat P, Nuñez R, Salem R (2008) Radioembolization of Yttrium-90 Microspheres for Hepatic Malignancy. Seminars in Interventional Radiology 25(1): 48-57. 
ISSN: 2574-1241

DOI: 10.26717/BJSTR.2018.09.001800

Jinendra Satiya. Biomed J Sci \& Tech Res

(c) (i) This work is licensed under Creative

Submission Link: https://biomedres.us/submit-manuscript.php

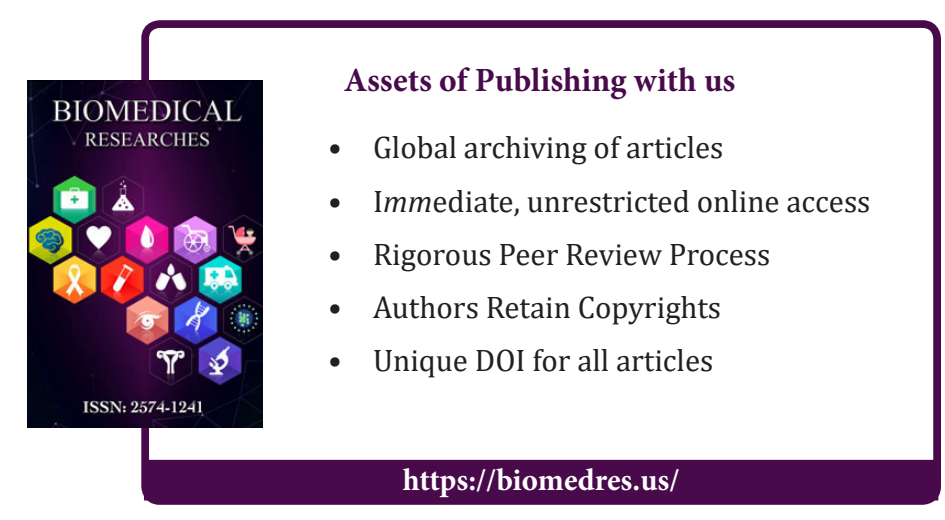

